\title{
NEW APPROACH TO SOLVE HYBRID FUZZY DIFFERENTIAL EQUATION
}

\author{
${ }^{1}$ Saveetha Natesan, ${ }^{2}$ Nirmala Venkatachalem and ${ }^{3}$ S. Chenthur Pandian \\ ${ }^{1}$ Department of Mathematics, Vivekananda College of Technology for Women, Tamil Nadu, India \\ ${ }^{2}$ Department of Mathematics, University College of Engineering Tindivanam, Tamil Nadu, India \\ ${ }^{3}$ Department of Electrical and Electronics Engineering, \\ Dr Mahalingam College of Engineering and Technology, Tamil Nadu, India
}

Received 2013-06-07, Revised 2013-06-24; Accepted 2013-07-01

\section{ABSTRACT}

This study presents solution for the first order hybrid fuzzy differential equation by Runge-Kutta method of order two with new parameters and Harmonic mean of $k_{\mathrm{i}}$ 's which are used in the main formula in order to increase the order of accuracy of the solution. This method is discussed in detail followed by a complete error analysis. The accuracy and efficiency of the proposed method is illustrated by solving a fuzzy initial value problem.

Keywords: Fuzzy Numbers, Fuzzy Differential Equations, Hybrid Systems, Multistep Runge-Kutta Method

\section{INTRODUCTION}

In recent years many works have been performed by several authors in numerical solutions of fuzzy differential equations. Fuzzy differential equations are applied in Population models, civil engineering, particle systems, medicine, bioinformatics and computational biology. Particularly, the use of Hybrid Fuzzy Differential equations is a natural way to model control systems with embedded uncertainty that are capable of controlling complex systems which have discrete event dynamics as well as continuous time dynamics. Furthermore there are some numerical techniques to solve hybrid fuzzy differential equations (Pederson and Sambandham, 2006; 2007; Prakash and Kalaiselvi, 2009; 2012; Ababneh et al., 2009; Nirmala and Pandian, 2011).

In this study, we develop numerical method for hybrid fuzzy differential equations by an application of the Runge-Kutta method of order two with new parameters and Harmonic mean of $\mathrm{k}_{\mathrm{i}}$ 's which are used in the main formula in order to increase the order of accuracy of the solution.

\section{PRELIMINARIES}

\section{Definition 2.1:}

A fuzzy number $\mathrm{u}$ is a fuzzy subset of $\mathrm{R}$ (i.e.,) $\mathrm{u}$ : $\mathrm{R} \rightarrow[0,1]$ satisfying the following conditions:

- $\quad \mathrm{u}$ is normal (i.e.,) $\exists \mathrm{x}_{0} \in \mathrm{R}$ with $\mathrm{u}\left(\mathrm{x}_{0}\right)=1$

- $\mathrm{u}$ is a convex fuzzy set (i.e.,) $\mathrm{u}(\lambda \mathrm{x}+(1-$ $\lambda) y) \geq \min \{u(x), u(y)\} \forall \lambda \in[0,1], x, y \in R$

- $\quad \mathrm{u}$ is upper semi continuous on $\mathrm{R}$

- $\{x \in R, u(x)>0\}$ is compact

Let $\mathrm{E}$ be the class of all fuzzy subsets of R. Then $\mathrm{E}$ is called the space of fuzzy numbers clearly, $\mathrm{R} \subset \mathrm{E}$ and $\mathrm{R} \subset \mathrm{E}$ is understood as $\mathrm{R}=\left\{\mathrm{R}_{\mathrm{x}}\right.$ : $\mathrm{R}$ is usual real number $\}$.

An arbitrary fuzzy number is represented by an ordered pair of functions $(\underline{\mathrm{u}}(\mathrm{r}), \overline{\mathrm{u}}(\mathrm{r})), 0 \leq \mathrm{r} \leq 1$ that satisfies the following requirements:

- $\underline{\mathrm{u}}(\mathrm{r})$ is a bounded left continuous non decreasing function over $[0,1]$, with respect to any $r$

Corresponding Author: Saveetha Natesan, Department of Mathematics, Vivekananda College of Technology for Women, Tamil Nadu, India 
- $\quad \overline{\mathrm{u}}(\mathrm{r})$ is a bounded right continuous non increasing function over $[0,1]$, with respect to any $r$

- $\quad \underline{\mathrm{u}}(\mathrm{r}) \leq \overline{\mathrm{u}}(\mathrm{r}), 0 \leq \mathrm{r} \leq 1$

Then the r-level set is $[u]_{r}=\{x \backslash u(x) \geq r\}, 0 \leq r \leq 1$ is a closed and bounded interval denoted by $[\mathrm{u}]_{\mathrm{r}}[\underline{\mathrm{u}}(\mathrm{r}), \overline{\mathrm{u}}(\mathrm{r})]$ and clearly, $[\mathrm{u}]_{0}=\{\mathrm{x} \mid \mathrm{u}(\mathrm{x})>0\}$ is compact.

\section{THE HYBRID FUZZY DIFFERENTIAL SYSTEM}

Consider the hybrid fuzzy differential system Equation (1):

$\left\{\begin{array}{l}x^{\prime}(t)=f\left(t, x(t), \lambda_{k}\left(x_{k}\right)\right), t \in\left[t_{k}, t_{k+1}\right] \\ x\left(t_{k}\right)=x_{k}\end{array}\right.$

where, $0 \leq \mathrm{t}_{0}<\mathrm{t}_{1}<\ldots<\mathrm{t}_{\mathrm{k}} \ldots \ldots \ldots \mathrm{t}_{\mathrm{k}} \rightarrow \infty$

$$
f \in C\left[R^{+} \times E \times E, E\right], \lambda_{k} \in C[E, E]
$$

Here, we assume that the existence and uniqueness of solution of the hybrid system hold on each $\left[\mathrm{t}_{\mathrm{k}}, \mathrm{t}_{\mathrm{k}+1}\right]$ to be specific the system would look like:

$$
\begin{aligned}
& x^{\prime}(t)= \\
& \left\{\begin{array}{l}
x_{0}{ }^{\prime}(t)=f\left(t, x_{0}(t), \lambda_{0}\left(x_{0}\right)\right), x\left(t_{0}\right)=x_{0}, t \in\left[t_{0}, t_{1}\right] \\
x_{1}{ }^{\prime}(t)=f\left(t, x_{1}(t), \lambda_{1}\left(x_{1}\right)\right), x\left(t_{1}\right)=x_{1}, t \in\left[t_{1}, t_{2}\right] \\
\vdots \\
x_{k}{ }^{\prime}(t)=f\left(t, x_{k}(t), \lambda_{k}\left(x_{k}\right)\right), x\left(t_{k}\right)=x_{k}, t \in\left[t_{k}, t_{k+1}\right] \\
\vdots
\end{array}\right.
\end{aligned}
$$

By the solution of (1) we mean the following function:

$$
x(t)=x\left(t, t_{0}, x_{0}\right)=\left\{\begin{array}{c}
x_{0}(t), t \in\left[t_{0}, t_{1}\right] \\
x_{1}(t), t \in\left[t_{1}, t_{2}\right] \\
\vdots \\
x_{k}(t), t \in\left[t_{k}, t_{k+1}\right] \\
\vdots
\end{array}\right.
$$

We note that the solutions of (1) are piecewise differentiable in each interval for $t \in\left[t_{k}, t_{k+1}\right]$ for a fixed $\mathrm{x}_{\mathrm{k}} \in \mathrm{E}$ and $\mathrm{k}=0,1,2, \ldots \ldots$
Using a representation of fuzzy numbers we may represent $\mathrm{x} \in \mathrm{E}$ by a pair of functions $(\underline{\mathrm{x}}(\mathrm{r}), \overline{\mathrm{x}}(\mathrm{r})), 0 \leq \mathrm{r} \leq 1$, such that:

- $\underline{\mathrm{x}}(\mathrm{r})$ is bounded, left continuous and non decreasing,

- $\bar{x}(r)$ is bounded, left continuous and non increasing and

- $\quad \underline{\mathrm{x}}(\mathrm{r}) \leq \overline{\mathrm{x}}(\mathrm{r}), 0 \leq \mathrm{r} \leq 1$

Therefore we may replace (1) by an equivalent system Equation (2):

$\left\{\begin{array}{l}\underline{\mathrm{x}}^{\prime}(\mathrm{t})=\underline{\mathrm{f}}\left(\mathrm{t}, \mathrm{x}, \lambda_{\mathrm{k}}\left(\mathrm{x}_{\mathrm{k}}\right)\right) \equiv \mathrm{F}_{\mathrm{k}}(\mathrm{t}, \underline{\mathrm{x}}, \overline{\mathrm{x}}), \underline{\mathrm{x}}\left(\mathrm{t}_{\mathrm{k}}\right)=\underline{\mathrm{x}}_{\mathrm{k}} \\ \overline{\mathrm{x}}^{\prime}(\mathrm{t})=\overline{\mathrm{f}}\left(\mathrm{t}, \mathrm{x}, \lambda_{\mathrm{k}}\left(\mathrm{x}_{\mathrm{k}}\right) \equiv \mathrm{G}_{\mathrm{k}}(\mathrm{t}, \underline{\mathrm{x}}, \overline{\mathrm{x}}), \overline{\mathrm{x}}\left(\mathrm{t}_{\mathrm{k}}\right)=\overline{\mathrm{x}}_{\mathrm{k}}\right.\end{array}\right.$

Which possesses a unique solution $(\underline{x}, \bar{x})$ which is a fuzzy function. That is for each $t, t$ he pair $[\underline{x}(t ; r), \bar{x}(t ; r)]$ is a fuzzy number, where $\underline{x}(t ; r), \bar{x}(t ; r)$ are respectively the solutions of the parametric form given by Equation (3):

$\left\{\begin{array}{l}\underline{x}^{\prime}(t)=F_{k}(t, \underline{x}(t ; r), \bar{x}(t ; r)), \underline{x}\left(t_{k} ; r\right)=\underline{x}_{k}(r) \\ \bar{x}^{\prime}(t)=G_{k}(t, \underline{x}(t ; r), \bar{x}(t ; r)), \bar{x}\left(t_{k} ; r\right)=\bar{x}_{k}(r)\end{array}\right.$

For $\mathrm{r} \in[0,1]$.

\section{THE SECOND ORDER RUNGE KUTTA METHOD WITH HARMONIC MEAN}

For a hybrid fuzzy differential equation we develop the second order Runge Kutta method with harmonic mean when $\mathrm{f}$ and $\lambda_{\mathrm{k}}$ in (1) can be obtained via the Zadeh (1965) extension principle from:

$$
\mathrm{f} \in \mathrm{c}\left[\mathrm{R}^{+} \times \mathrm{R} \times \mathrm{R}, \mathrm{R}\right] \text { and } \lambda_{\mathrm{k}} \in \mathrm{C}[\mathrm{R}, \mathrm{R}]
$$

We assume that the existence and uniqueness of solutions of (1) hold for each $\left[t_{k}, t_{k+1}\right]$. For a fixed $r$, to integrate the system in (3) $\left[\mathrm{t}_{0}, \mathrm{t}_{1}\right],\left[\mathrm{t}_{1}, \mathrm{t}_{2}\right] \ldots \ldots$ $\ldots\left[\mathrm{t}_{\mathrm{k}}, \mathrm{t}_{\mathrm{k}+1}\right] \ldots$... we replace each interval by a set of $\mathrm{N}_{\mathrm{k}+1}$ discrete equally spaced grid points (including the end points) at which the exact solution $\mathrm{x}(\mathrm{t} ; \mathrm{r})=(\underline{\mathrm{x}}(\mathrm{t} ; \mathrm{r}), \overline{\mathrm{x}}(\mathrm{t} ; \mathrm{r})) \quad$ is approximated by some $\left(\underline{y}_{k}(t ; r), \bar{y}_{k}(t ; r)\right)$. For the chosen grid points on $\left[t_{k}, t_{k+1}\right]$ at $\mathrm{t}_{\mathrm{k}, \mathrm{n}}=\mathrm{t}_{\mathrm{k}}+\mathrm{nh}_{\mathrm{k}}, \mathrm{h}_{\mathrm{k}}=\frac{\mathrm{t}_{\mathrm{k}+1}-\mathrm{t}_{\mathrm{k}}}{\mathrm{N}_{\mathrm{k}}}, 0 \leq \mathrm{n} \leq \mathrm{N}_{\mathrm{k}}$. Let 
$\left(\underline{\mathrm{Y}}_{\mathrm{k}}(\mathrm{t} ; \mathrm{r}), \overline{\mathrm{Y}}_{\mathrm{k}}(\mathrm{t} ; \mathrm{r})\right) \equiv(\underline{\mathrm{x}}(\mathrm{t} ; \mathrm{r}), \overline{\mathrm{x}}(\mathrm{t} ; \mathrm{r})),\left(\underline{\mathrm{y}}_{\mathrm{k}}(\mathrm{t} ; \mathrm{r}), \overline{\mathrm{y}}_{\mathrm{k}}(\mathrm{t} ; \mathrm{r})\right) \quad$ and $\left(\underline{\mathrm{y}}_{\mathrm{k}}(\mathrm{t} ; \mathrm{r}), \overline{\mathrm{y}}_{\mathrm{k}}(\mathrm{t} ; \mathrm{r})\right)$ may be denoted respectively by $\left(\underline{\mathrm{Y}}_{k, \mathrm{n}}(\mathrm{r}), \overline{\mathrm{Y}}_{\mathrm{k}, \mathrm{n}}(\mathrm{r})\right)$ and $\left(\underline{\mathrm{y}}_{\mathrm{k}, \mathrm{n}}(\mathrm{r}), \overline{\mathrm{y}}_{\mathrm{k}, \mathrm{n}}(\mathrm{r})\right)$. We allow the $\mathrm{N}_{\mathrm{k}}$ 's to vary over the $\left[t_{k}, t_{k+1}\right]$ 's so that the $h_{k}$ 's may be comparable. The second order Runge Kutta method for (1) is given by:

$$
\begin{gathered}
\underline{y_{k, n+1}}(r)-\underline{y_{k, n}}(r)=2 \frac{k_{1}\left(t_{k, n} ; y_{k, n}(r)\right) \underline{k_{2}}\left(t_{k, n} ; y_{k, n}(r)\right)}{\underline{k_{1}}\left(t_{k, n} ; y_{k, n}(r)\right)+\underline{k_{2}}\left(t_{k, n} ; y_{k, n}(r)\right)} \\
\overline{y_{k, n+1}}(r)-\overline{y_{k, n}}(r)=2 \frac{\overline{k_{1}}\left(t_{k, n} ; y_{k, n}(r)\right) \overline{k_{2}}\left(t_{k, n} ; y_{k, n}(r)\right)}{\overline{k_{1}}\left(t_{k, n} ; y_{k, n}(r)\right)+\overline{k_{2}}\left(t_{k, n} ; y_{k, n}(r)\right)}
\end{gathered}
$$

Where:

$$
\begin{aligned}
& \underline{k_{1}}\left(t_{k, n} ; y_{k, n}(r)\right)=\min \left\{\begin{array}{l}
h_{k} f\left(t_{k, n}, u, \lambda_{k}\left(u_{k}\right)\right) \backslash \\
u \in\left[y_{k, n}(r), \overline{y_{k, n}}(r)\right], \\
u_{k} \in\left[\underline{y_{k, 0}}(r), \overline{y_{k, 0}}(r)\right]
\end{array}\right\}, \\
& \overline{\mathrm{k}_{1}}\left(\mathrm{t}_{\mathrm{k}, \mathrm{n}} ; \mathrm{y}_{\mathrm{k}, \mathrm{n}}(\mathrm{r})\right)=\max \left\{\begin{array}{l}
\mathrm{h}_{\mathrm{k}} \mathrm{f}\left(\mathrm{t}_{\mathrm{k}, \mathrm{n}}, \mathrm{u}, \lambda_{\mathrm{k}}\left(\mathrm{u}_{\mathrm{k}}\right)\right) \\
\mathrm{lu} \in\left[\underline{\mathrm{y}_{\mathrm{k}, \mathrm{n}}}(\mathrm{r}), \overline{\mathrm{y}_{\mathrm{k}, \mathrm{n}}}(\mathrm{r})\right], \\
\mathrm{u}_{\mathrm{k}} \in\left[\underline{\underline{\mathrm{y}_{k, 0}}}(\mathrm{r}), \overline{\mathrm{y}_{\mathrm{k}, 0}}(\mathrm{r})\right]
\end{array}\right\},
\end{aligned}
$$

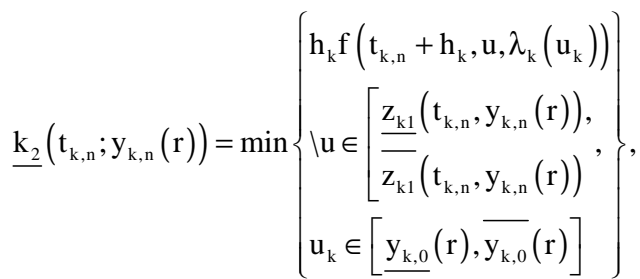

$$
\begin{aligned}
& \overline{\mathrm{k}_{2}}\left(\mathrm{t}_{\mathrm{k}, \mathrm{n}} ; \mathrm{y}_{\mathrm{k}, \mathrm{n}}(\mathrm{r})\right)=\max \left\{\begin{array}{c}
\mathrm{h}_{\mathrm{k}} \mathrm{f}\left(\mathrm{t}_{\mathrm{k}, \mathrm{n}}+\mathrm{h}_{\mathrm{k}}, \mathrm{u}, \lambda_{\mathrm{k}}\left(\mathrm{u}_{\mathrm{k}}\right)\right) \\
\mathrm{lu} \in\left[\begin{array}{l}
\mathrm{z}_{\mathrm{k} 1}\left(\mathrm{t}_{\mathrm{k}, \mathrm{n}}, \mathrm{y}_{\mathrm{k}, \mathrm{n}}(\mathrm{r})\right), \\
\overline{\mathrm{z}_{\mathrm{k} 1}}\left(\mathrm{t}_{\mathrm{k}, \mathrm{n}}, \mathrm{y}_{\mathrm{k}, \mathrm{n}}(\mathrm{r})\right), \\
\mathrm{u}_{\mathrm{k}} \in\left[\mathrm{y}_{\mathrm{k}, 0}(\mathrm{r}), \overline{\mathrm{y}_{\mathrm{k}, 0}}(\mathrm{r})\right]
\end{array}\right.
\end{array}\right\}
\end{aligned}
$$

Where:

$$
\underline{\mathrm{z}_{\mathrm{k} 1}}\left(\mathrm{t}_{\mathrm{k}, \mathrm{n}}, \mathrm{y}_{\mathrm{k}, \mathrm{n}}(\mathrm{r})\right)=\underline{\mathrm{y}_{\mathrm{k}, \mathrm{n}}}(\mathrm{r})+\underline{\mathrm{k}_{1}}\left(\mathrm{t}_{\mathrm{k}, \mathrm{n}}, \mathrm{y}_{\mathrm{k}, \mathrm{n}}(\mathrm{r})\right)
$$

$$
\overline{\mathrm{z}_{\mathrm{k} 1}}\left(\mathrm{t}_{\mathrm{k}, \mathrm{n}}, \mathrm{y}_{\mathrm{k}, \mathrm{n}}(\mathrm{r})\right)=\overline{\mathrm{y}_{\mathrm{k}, \mathrm{n}}}(\mathrm{r})+\overline{\mathrm{k}_{1}}\left(\mathrm{t}_{\mathrm{k}, \mathrm{n}}, \mathrm{y}_{\mathrm{k}, \mathrm{n}}(\mathrm{r})\right)
$$

Next we define:

$$
\begin{aligned}
& S_{k}\left[t_{k, n}, \underline{y_{k, n}}(r), \overline{y_{k, n}}(r)\right] \\
& =2 \frac{\underline{k_{2}}\left(t_{k, n} ; y_{k, n}(r)\right) \underline{k_{z}}\left(t_{k, n} ; y_{k, n}(r)\right)}{\underline{k_{2}}\left(t_{k, n} ; y_{k, n}(r)\right)+\underline{k_{z}}\left(t_{k, n} ; y_{k, n}(r)\right)} \\
& T_{k}\left[t_{k, n}, \underline{y_{k, n}}(r), \overline{y_{k, n}}(r)\right] \\
& =2 \frac{\overline{k_{2}}\left(t_{k, n} ; y_{k, n}(r)\right) \overline{k_{z}}\left(t_{k, n} ; y_{k, n}(r)\right)}{\overline{k_{2}}\left(t_{k, n} ; y_{k, n}(r)\right)+\overline{k_{z}}\left(t_{k, n} ; y_{k, n}(r)\right)}
\end{aligned}
$$

The exact solution at $\mathrm{t}_{\mathrm{k}, \mathrm{n}+1}$ is given by:

$$
\left\{\begin{array}{l}
\underline{\mathrm{Y}_{\mathrm{k}, \mathrm{n}+1}}(\mathrm{r}) \approx \underline{\mathrm{Y}_{\mathrm{k}, \mathrm{n}}}(\mathrm{r})+\mathrm{S}_{\mathrm{k}}\left[\mathrm{t}_{\mathrm{k}, \mathrm{n}}, \underline{\mathrm{y}_{\mathrm{k}, \mathrm{n}}}(\mathrm{r}), \overline{\mathrm{y}_{\mathrm{k}, \mathrm{n}}}(\mathrm{r})\right] \\
\overline{\mathrm{Y}_{\mathrm{k}, \mathrm{n}+1}}(\mathrm{r}) \approx \overline{\mathrm{Y}_{\mathrm{k}, \mathrm{n}}}(\mathrm{r})+\mathrm{T}_{\mathrm{k}}\left[\mathrm{t}_{\mathrm{k}, \mathrm{n}}, \underline{\mathrm{y}_{\mathrm{k}, \mathrm{n}}}(\mathrm{r}), \overline{\mathrm{y}_{\mathrm{k}, \mathrm{n}}}(\mathrm{r})\right]
\end{array}\right.
$$

The approximate solution is given by Equation (4):

$$
\left\{\begin{array}{l}
\overline{\mathrm{Y}_{\mathrm{k}, \mathrm{n}+1}}(\mathrm{r}) \approx \overline{\mathrm{Y}_{\mathrm{k}, \mathrm{n}}}(\mathrm{r})+\mathrm{S}_{\mathrm{k}}\left[\mathrm{t}_{\mathrm{k}, \mathrm{n}}, \underline{\mathrm{y}_{\mathrm{k}, \mathrm{n}}}(\mathrm{r}) \overline{\mathrm{y}_{\mathrm{k}, \mathrm{n}}}(\mathrm{r})\right] \\
\overline{\mathrm{Y}_{\mathrm{k}, \mathrm{n}+1}}(\mathrm{r}) \approx \overline{\mathrm{Y}_{\mathrm{k}, \mathrm{n}}}(\mathrm{r})+\mathrm{T}_{\mathrm{k}}\left[\mathrm{t}_{\mathrm{k}, \mathrm{n}}, \underline{\mathrm{y}_{\mathrm{k}, \mathrm{n}}}(\mathrm{r}) \overline{\mathrm{y}_{\mathrm{k}, \mathrm{n}}}(\mathrm{r})\right]
\end{array}\right.
$$

\section{Lemma 4.1}

Suppose $i \in Z^{+}, \varepsilon_{i}>0, r \in[0,1]$ and $h_{i}<1$ are fixed. Let $\left\{\mathrm{Z}_{\mathrm{i}, \mathrm{n}}(\mathrm{r})\right\}_{\mathrm{n}=0}^{\mathrm{N}_{\mathrm{i}}}$ be the Runge Kutta like formulae approximation with $\mathrm{N}=\mathrm{N}_{\mathrm{i}}$ to the fuzzy IVP equation (5):

$\left\{\begin{array}{l}x^{\prime}(t)=f\left(t, x(t), \lambda_{i}\left(x_{i}\right)\right), t \in\left[t_{i}, t_{i+1}\right] \\ x\left(t_{i}\right)=x_{i}\end{array}\right.$

If $\left\{\mathrm{y}_{\mathrm{i}, \mathrm{n}}(\mathrm{r})\right\}_{\mathrm{n}=0}^{\mathrm{N}_{\mathrm{i}}}$ denotes the result (4) from some $\mathrm{y}_{\mathrm{i}, 0}(\mathrm{r})$, then there exists a $\delta_{\mathrm{i}}>0$ such that:

$$
\begin{aligned}
& \left|\underline{z}_{i, 0}(r)-\underline{y}_{i, 0}(r)<\delta_{i},\right| \bar{z}_{i, 0}(r)-\bar{y}_{i, 0}(r)<\delta_{i} \text { implies } \\
& \left|\underline{z}_{i, N i}(r)-\underline{y}_{i, N i}(r)\right|<\varepsilon_{i}, \mid \bar{z}_{i, N i}(r)-\bar{y}_{i, N i}(r)<\varepsilon_{i}
\end{aligned}
$$




\section{Theorem 4.1}

Consider the systems (2) and (4). For a fixed $k \in Z^{+}$ and $\mathrm{r} \in[0,1]$ :

$$
\begin{aligned}
& \lim _{\mathrm{h}_{\mathrm{o}} \ldots \mathrm{h}_{\mathrm{k}} \rightarrow 0} \underline{y}_{k, N \mathrm{Nk}}(\mathrm{r})=\underline{\mathrm{x}}\left(\mathrm{t}_{\mathrm{k}+1} ; \mathrm{r}\right), \\
& \lim _{\mathrm{h}_{\mathrm{o} \ldots \ldots \mathrm{h}_{\mathrm{k}} \rightarrow 0}} \bar{y}_{\mathrm{k}, \mathrm{Nk}}(\mathrm{r})=\overline{\mathrm{x}}\left(\mathrm{t}_{\mathrm{k}+1} ; \mathrm{r}\right)
\end{aligned}
$$

\section{NUMERICAL ILLUSTRATION}

To give a clear overview of our study and to illustrate the above discussed technique, we consider the following examples.

\section{Example 5.1}

Consider the following hybrid fuzzy IVP:

$$
\left\{\begin{array}{l}
x^{\prime}(t)=x(t)+m(t) \lambda_{k}\left(x\left(t_{k}\right)\right), t \in\left[t_{k}, t_{k+1}\right] \\
t_{k}=k, k=0,1,2, . \\
x(0, r)=[0.75+0.25 r, 1.125-0.125 r] 0 \leq r \leq 1
\end{array}\right.
$$

Where:

$$
\begin{gathered}
\mathrm{m}(\mathrm{t})=\left\{\begin{array}{cc}
2(\mathrm{t}(\bmod 1)), & \text { if } \mathrm{t}(\bmod 1) \leq 0.5 \\
2(1-\mathrm{t}(\bmod 1)), & \text { if } \mathrm{t}(\bmod 1)>0.5
\end{array}\right. \\
\lambda_{\mathrm{k}}(\mu)= \begin{cases}\hat{0}, & \text { if } \mathrm{k}=0 \\
\mu, & \text { if } \mathrm{k} \in\{1,2, \ldots \ldots .\}\end{cases}
\end{gathered}
$$

The exact solution for $t \in[0,1]$ is:

$$
x(t ; r)=\left[(0.75+0.25 r) e^{t},(1.125-0.125 r) e^{t}\right] .
$$

For $\mathrm{t} \in[1,2]$, the exact solution satisfies:

$$
x(t ; r)=x(1, r)\left[2 t-2+e^{t-1.5}(3 \sqrt{e}-4)\right]
$$

Then $\mathrm{x}(2.0,1)$ is approximately 9.68 and $\mathrm{y}_{1,10}(1)$ is approximately 9.6031. For additional comparison, Fig. 1 shows the graphs of $\mathrm{x}(2.0), \mathrm{y}_{1,10}$ and the corresponding Euler approximation.

Consider the following hybrid fuzzy IVP:

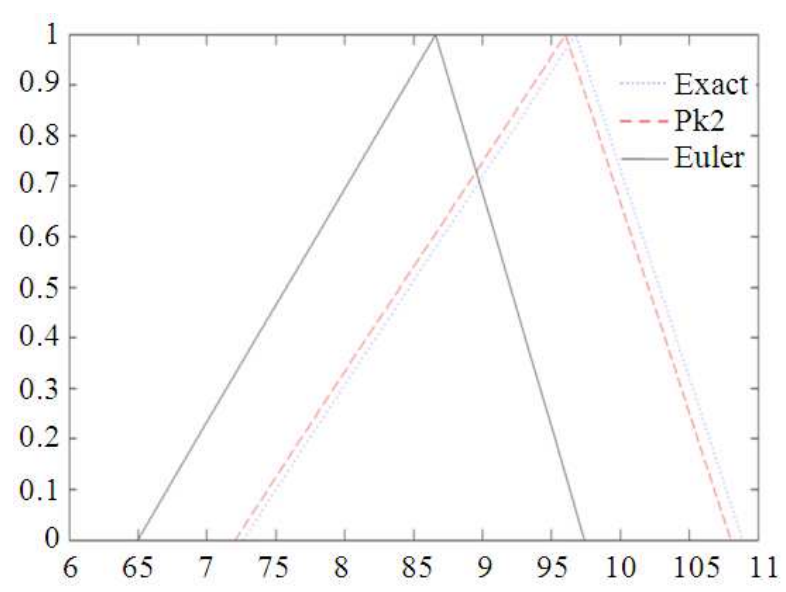

Fig. 1. Comparison of exact and approximated solutions of Example 1

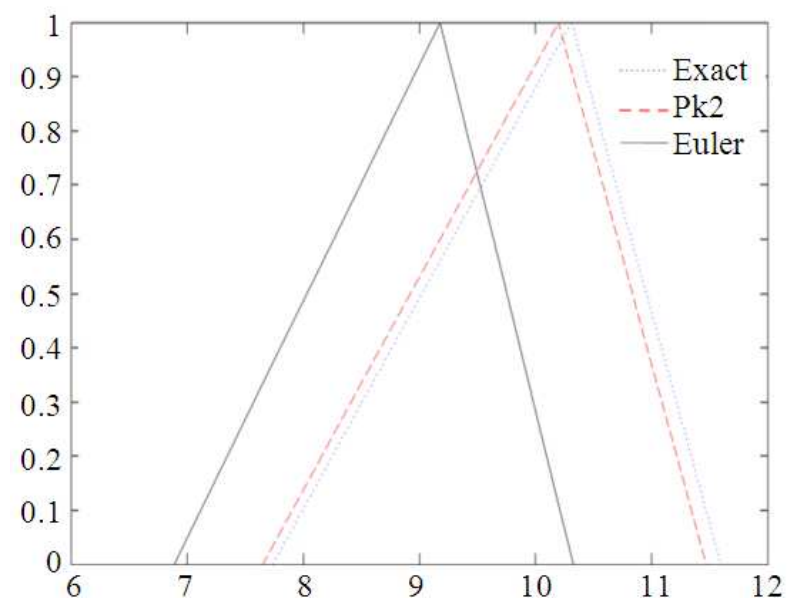

Fig. 2. Comparison of exact and approximated solutions of Example 1

$x^{\prime}(t)=x(t)+m(t) \lambda_{k}\left(x\left(t_{k}\right)\right), t \in\left[t_{k}, t_{k+1}\right], t_{k}=k, k=0,1,2$, $\mathrm{x}(0, \mathrm{r})=[0.75+0.25 \mathrm{r}, 1.125-0.125 \mathrm{r}] 0 \leq \mathrm{r} \leq 1$.

where, $\mathrm{m}(\mathrm{t})=|\sin (\pi \mathrm{t})|, \mathrm{k}=0,12, \ldots \ldots$ :

$\lambda_{\mathrm{k}}(\mu)=\left\{\begin{array}{lc}\hat{0}, \quad \text { if } \mathrm{k}=0 \\ \mu, \quad \text { if } \mathrm{k} \in\{1,2, \ldots \ldots\}\end{array}\right.$

The exact solution for $t \in[0,1]$ is:

$x(t ; r)=\left[(0.75+0.25 r) e^{t},(1.125-0.125 r) e^{t}\right]$ 
For $\mathrm{t} \in[1,2]$, the exact solution satisfies:

$x(t ; r)=x(1, r) \frac{\pi \cos (\pi t)+\sin (\pi t)}{\pi^{2}+1}+\frac{e^{t}}{e} x(1, r)\left(1+\frac{\pi}{\pi^{2}+1}\right)$

Then $\mathrm{x}(2.0,1)$ is approximately 10.31 and $\mathrm{y}_{1,10}(1)$ is approximately 10.198. For additional comparison, Fig. 2 shows the graphs of $\mathrm{x}(2.0), \mathrm{y}_{1}, 1_{10}$ and the corresponding Euler approximation.

\section{CONCLUSION}

In this study we applied Runge Kutta method of order two for finding the numerical solution of hybrid fuzzy differential equations. Taking into account the convergence order of the Euler method is $0(\mathrm{~h})$, a higher order of convergence $0\left(h^{3}\right)$ is obtained by the proposed method.

\section{REFERENCES}

Ababneh, O.Y., Ahmad, R. and E.S. Ismail, 2009. New multi step Runge Kutta method. Applied Math. Sci., 3: 2255-2262.
Nirmala, V. and S.C. Pandian, 2011. New multi-step Runge-Kutta method for solving fuzzy differential equations. Math. Theory Model., 1: 16-22.

Pederson, S. and M. Sambandham, 2006. The RungeKutta method for hybrid fuzzy differential equations. Nonlinear Anal. Hybrid Syst., 2: 626-634. DOI:10.1016/j.nahs.2006.10.013

Pederson, S. and M. Sambandham, 2007. Numerical solution to hybrid fuzzy systems. Math. Comput. Model., 45: 1133-1144. DOI: 10.1016/j.mcm.2006.09.014

Prakash, P. and V. Kalaiselvi, 2009. Numerical solution of hybrid fuzzy differential equations by predictorcorrector method. Int. J. Comput. Math., 86: 121134. DOI: $10.1080 / 00207160802247620$

Prakash, P. and V. Kalaiselvi, 2012. Numerical solutions of fuzzy differential equations by using hybrid methods. Fuzzy Inform. Eng., 4: 445-455. DOI: 10.1007/s12543-012-0126-9

Zadeh, L.A., 1965. Fuzzy sets. Inform. Control, 8: 338353. DOI: 10.1016/S0019-9958(65)90241-X 